\title{
TITLE:
}

\section{Crucial importance of translational entropy of water in pressure denaturation of proteins}

$\operatorname{AUTHOR}(\mathrm{S})$ :

Harano, Y; Kinoshita, M

\section{CITATION:}

Harano, Y ... [et al]. Crucial importance of translational entropy of water in pressure denaturation of proteins. The Journal of Chemical Physics 2006, 125(2): 024910.

\section{ISSUE DATE:}

2006-07-14

URL:

http://hdl.handle.net/2433/50435

\section{RIGHT:}

Copyright 2006 American Institute of Physics. This article may be downloaded for personal use only. Any other use requires prior permission of the author and the American Institute of Physics. 


\title{
Crucial importance of translational entropy of water in pressure denaturation of proteins
}

\author{
Yuichi Harano and Masahiro Kinoshita ${ }^{\text {a) }}$ \\ International Innovation Center, Kyoto University, Uji, Kyoto 611-0011, Japan
}

(Received 17 April 2006; accepted 30 May 2006; published online 14 July 2006)

\begin{abstract}
We present statistical thermodynamics of pressure denaturation of proteins, in which the three-dimensional integral equation theory is employed. It is applied to a simple model system focusing on the translational entropy of the solvent. The partial molar volume governing the pressure dependence of the structural stability of a protein is expressed for each structure in terms of the excluded volume for the solvent molecules, the solvent-accessible surface area (ASA), and a parameter related to the solvent-density profile formed near the protein surface. It is argued that the entropic effect originating from the translational movement of water molecules plays critical roles in the pressure-induced denaturation. We also show that the exceptionally small size of water molecules among dense liquids in nature is crucial for pressure denaturation. An unfolded structure, which is only moderately less compact than the native structure but has much larger ASA, is shown to turn more stable than the native one at an elevated pressure. The water entropy for the native structure is higher than that for the unfolded structure in the low-pressure region, whereas the opposite is true in the high-pressure region. Such a structure is characterized by the cleft and/or swelling and the water penetration into the interior. In another solvent whose molecular size is 1.5 times larger than that of water, however, the inversion of the stability does not occur any longer. The random coil becomes relatively more destabilized with rising pressure, irrespective of the molecular size of the solvent. These theoretical predictions are in qualitatively good agreement with the experimental observations. (c) 2006 American Institute of Physics. [DOI: 10.1063/1.2217011]
\end{abstract}

\section{INTRODUCTION}

A protein spontaneously folds into a unique native structure and becomes functional in aqueous solution under physiological conditions. ${ }^{1}$ However, the native structure is unfolded by various perturbations such as the addition of chemical substances, the change in the temperature or $p \mathrm{H}$, and the application of a high pressure. ${ }^{2-6}$ Recent experimental studies indicate that the ensemble of unfolding pathways for pressure denaturation is inherently different from those for heat or chemical denaturation. ${ }^{3,4}$ This implies that a specific viewpoint is necessary for uncovering the mechanism of pressure denaturation. The pressure-induced unfolding, which should be related to the pressure effects on various physicochemical processes occurring in aqueous solutions, presents much challenge in chemical physics as well as in biophysics and biochemistry.

The pressure-denatured structures are characterized by the swelling, water penetration into the interior, and only a moderate reduction of the compactness. ${ }^{2-7}$ They are in contrast with the random coils. One of the most essential experimental results for pressure denaturation is the crucial importance of water. ${ }^{8}$ Adding glycerol as a cosolvent to water increases the pressure required for denaturation of the Arc repressor protein. An extrapolation to a pure glycerol solvent suggests that the Arc repressor protein could not be pressure denatured in glycerol. It is therefore concluded that water is

\footnotetext{
${ }^{a)}$ Author to whom correspondence should be addressed. Fax: +81-774-383508. Electronic mail: kinoshit@iae.kyoto-u.ac.jp
}

crucial for pressure denaturation. It is interesting to ask the following question: How does water differ from the other solvents in terms of the pressure effect on the structural stability of a protein?

The mechanism of pressure denaturation is often discussed in terms of the pressure dependence of the hydrophobicity of small molecules or protein subunits. For the methane-methane interaction in water, the stability of the contact pair relative to the water-separated one is shown to decrease with rising pressure. ${ }^{9}$ Computer simulation studies $^{7,10,11}$ indicate that nonpolar side chains are more separated in a pressure-denatured structure with water molecules penetrating its hydrophobic core than in the native one. Based on this indication it is concluded that the weakening of the hydrophobic interactions between nonpolar side chains is the major cause of pressure denaturation. However, the physical origin of the weakening is rather unclear. (We give a comment on the concept of the weakening in Sec. IV.) For a complete interpretation of the protein denaturation, it is necessary to treat a protein itself immersed in water rather than to deduce the mechanism from the behavior of small molecules or protein subunits.

Recently, we have shown that the entropic effect, which promotes an increase in the translational entropy of water (e.g., a reduction of the excluded volume leading to an increase in the total volume available to the translational movement of water molecules that are present in the whole system), is a major driving force in protein folding under physiological conditions. ${ }^{12,13}$ In vacuum, the formation of 
intramolecular hydrogen bonds and salt bridges leads to great energy gain and stability. However, the formation in water is accompanied by the serious energetic penalty of dehydration. This is one of the reasons for the dominance of the water entropy. More details are described in our earlier publications. ${ }^{12,13}$ A major concern of this article is to prove that the entropic effect also plays crucially important roles in pressure denaturation of proteins and that pressure denaturation can hardly occur in solvents other than water.

The structural stability of a protein is determined by the free energy of the protein-aqueous solution system. The free energy can be argued in terms of the intramolecular energy and the conformational entropy of the protein and its hydration free energy (i.e., the excess chemical potential of the protein). Here, we postulate that the native structure is the most stable at a low pressure. A question is raised: What is the major cause of the structural change occurring at a high pressure? At least within the framework of classical mechanics, the intramolecular energy for any protein structure remains unchanged. The conformational entropy of an unfolded state is higher than that of the native state, but the difference in this quantity between the two states is thought to be independent of the pressure. It follows that the change in the hydration free energy is the key to the question raised above.

The hydration free energy consists of two components which are, respectively, energetic and entropic in origin. From the energetic viewpoint, the penetration of water molecules into the hydrophobic core of a protein should be rather unfavorable due to the loss of water-water hydrogen bonds. As for the entropic contribution to the hydration free energy of a solute, it originates from the excluded-volume effect (effect 1) referred to above and the effect due to the density and orientational structure of water molecules near the solute (effect 2). Effect 2 is dependent on the electrostatic and van der Waals solute-water interactions while effect 1 is not. The restriction of the translational movement of water molecules contributes to both of the effects while that of the rotational motion contributes only to effect $2 .^{14}$ It has been shown, with realistic water models, that for a large solute such as a protein effect 1 dominates and can be evaluated by modeling water molecules as hard spheres as long as the size of the hard spheres is set equal to that of water molecules. ${ }^{15}$ Even for effect 2, it has been shown that the translational contribution to the hydration entropy is much more important than the orientational one when the solute is sufficiently large. ${ }^{16,17}$ The qualitative aspects of the translational contribution can be studied by the hard-sphere model for water. $^{12,13}$ We note that the translational-entropy effect, which is a major cause of the solute hydrophobicity, is strongly dependent on the pressure ${ }^{12,13}$ and likely to play dominant roles in the change in the hydration free energy which leads to pressure denaturation. One might think that the native structure with a very small excluded volume, in which the backbone and side chains are closely packed with little space in the interior, ${ }^{18,19}$ is further stabilized through the entropic effect by applying a higher pressure. We show that this thought is incorrect for a protein with the complex polyatomic structure.
In this article, we wish to reveal the essential physics giving rise to pressure denaturation of proteins and elucidating the criticalness of water in the denaturation. To this end, we adopt a simplified model focusing on the entropic effect caused by the solvent. The model is simple in the sense that the electrostatic and van der Waals interactions are excluded. However, it accurately accounts for the complex polyatomic structure of a protein. With computer simulations using allatom potentials, which have been employed by many research groups, it is hard to interpret the results obtained and extract the essential physics. We employ an elaborate statistical-mechanical theory for liquids and show that pressure denaturation can be elucidated in terms of the purely entropic effect for water. Based on the thermodynamic argument combined with the theory, we point out that a pressuredenatured structure is rather compact but has a very large water-accessible surface area. It is also shown that the exceptionally small size of water molecules among ordinary liquids in nature ${ }^{20}$ is essential in causing pressure denaturation. A striking result is that the entropic effect, which greatly stabilizes the native structure at a low pressure, drives the protein to be denatured at a sufficiently high pressure. The features of the pressure-denatured structure theoretically predicted are in qualitatively good accord with the experimental observations. A brief report (letter to the editor) of this study is published in Ref. 21 but this article presents much more detailed discussions with lots of new calculation results.

\section{THEORETICAL ANALYSIS ON PRESSURE DENATURATION OF PROTEINS}

\section{A. Statistical thermodynamics of pressure denaturation}

The important thermodynamic quantities in discussing pressure denaturation are the solvation free energy (SFE) $\Delta \mu$ (i.e., the excess chemical potential) and the partial molar volume (PMV) $v$. The SFE governs the solvation properties of a protein and the PMV describes the pressure dependence of the SFE. We employ the three-dimensional (3D) integral equation theory ${ }^{22,23}$ to account for the complex polyatomic structure of a protein. The SFE and PMV are considered for a prescribed structure. The details of this theory and the numerical procedure for solving the basic equations ${ }^{12,13,23,24}$ are described in the Appendix. The SFE is calculated by simple integrations of the protein-solvent correlation functions and products thereof [see Eq. (A4) in the Appendix]. ${ }^{12,13,24}$ The PMV is calculated in accordance with the formulation, ${ }^{25}$

$$
v=\iiint\{1-g(x, y, z)\} d x d y d z,
$$

where $g(x, y, z)$ represents the microstructure of the solvent near the protein surface and is referred to as the reduced density profile. It has the physical meaning that the number of solvent molecules within the volume element $d x d y d z$ is given by $\rho g(x, y, z) d x d y d z$ ( $\rho$ is the solvent number density in the bulk). The ideal term $\chi_{T} k_{B} T$ ( $\chi_{T}$ is the isothermal compressibility of pure solvent and $k_{B}$ is Boltzmann's constant), which is relatively much smaller, is not included in the right hand of Eq. (1). The ideal term makes no contribution to the 


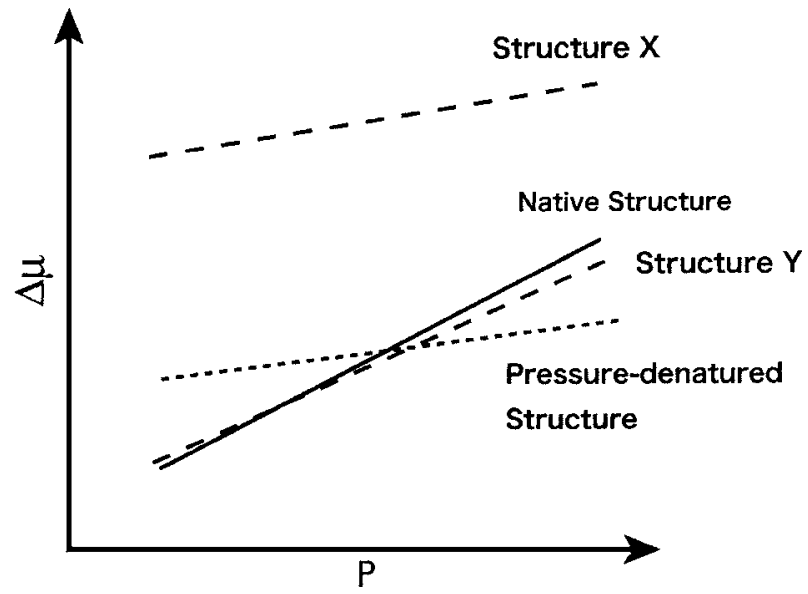

FIG. 1. Schematic relation between the solvation free energy $\Delta \mu$ and the pressure $P$ for three different structures of a protein. The slope represents the partial molar volume (PMV). Though the straight lines are drawn for simplicity, the actual PMV is not constant [see Fig. 3(b)].

relative values of the PMV among different protein structures in which we are interested.

The PMV is the pressure derivative of the SFE,

$$
v=(\partial \Delta \mu / \partial P)_{T},
$$

where $P$ and $T$ denote the pressure and absolute temperature, respectively. Figure 1 illustrates a schematic relation between $\Delta \mu$ and $P$ for three different structures including the native structure and a pressure-denatured one. The slope is the PMV defined by Eq. (2). For the denaturation to occur, " $\Delta \mu$ of the denatured structure minus $\Delta \mu$ of the native structure" must decrease to a significant extent as $P$ increases, requiring that the PMV of the denatured structure be sufficiently smaller than that of the native one. As a striking case, although the SFE of the denatured structure is higher than that of the native one in the low-pressure region, the inversion in the SFE occurs at a sufficiently high pressure, as shown in Fig. 1. We show in this article that the inversion actually occurs through the water-entropy change. Even when there is a structure (an example is structure $\mathrm{X}$ in the figure) whose PMV is smaller than that of the native one, however, such inversion can hardly occur if its SFE is much higher. Therefore, the SFE of the denatured structure needs to be sufficiently low even at low pressures. A structure like structure $\mathrm{Y}$ in the figure, which is only slightly different from the native structure and has correspondingly smaller PMV, is not considered as a pressure-denatured one. Such a structure is much less stable than the pressure-denatured one at high pressures.

\section{B. Model}

The statistical thermodynamics described above can be applied to any model of the protein-aqueous solution system. To focus on the entropic effect in our analysis, however, the solvent molecules are modeled as hard spheres with diameter $d=0.28 \mathrm{~nm}$ that is the size of water molecules, and a protein molecule is treated as a set of fused hard spheres. The complex polyatomic structure, which is critical in analyzing pres- sure denaturation, is taken into account at the atomic level. The diameter of each atom is set at the Lennard-Jones diameter (the $\sigma$ value) of AMBER 99. In this model system, all the allowed configurations share the same energy, and the system behavior is purely entropic in origin. As discussed in Sec. I, the SFE is the key quantity in pressure denaturation because its component related to the translational entropy of the solvent is most strongly dependent on the pressure. The SFE, which is attributable to the entropic effect alone in our model, governs the structural stability of a protein for changing pressure (the solvation entropy under the isochoric condition $\Delta S$ is exactly equal to $-\Delta \mu / T$, where $T$ is the absolute temperature).

\section{Geometric measures of protein structure and partial molar volume}

We now divide the term in the right hand of Eq. (1) into the integrations inside and outside the core region. Inside the core region, due to the overlap of the protein and solvent, the protein-solvent potential is infinitely large and $g=0$. It follows that the integration inside the core region equals the excluded volume (EV) which the centers of solvent molecules cannot enter (the $\mathrm{EV}$ is denoted by $v_{\mathrm{ex}}$ ). We remark that the EV includes void spaces in the protein interior. The $\mathrm{EV}$ can be a measure of the compactness of the protein structure. It tends to be smaller for a more compact structure. The integration outside the core region takes a negative value because a layer within which the solvent density is higher than in the bulk is formed near the protein surface due to the packing force arising from the translational movement of solvent molecules. Since the higher density is almost limited to the first layer (i.e., the thickness of the denser layer reaches only about half of the solvent diameter), the integration outside the core region is roughly in proportion to the solvent-accessible surface area (ASA) denoted by $a$ and the solvent diameter $d$. Thus, we can write

$$
v \sim v_{\mathrm{ex}}-\xi d a, \quad \xi>0 .
$$

The parameter $\xi$ is related to the average solvent density within the dense layer. The formulation conveniently describes the PMV in terms of $\xi$ and only the two geometric measures, $v_{\mathrm{ex}}$ and $a$. In the present analysis, $v_{\mathrm{ex}}$ and $a$ are calculated using an analytical method. ${ }^{26}$ We note that the formulation is different from that given in Ref. 21. The present one accounts for the effect due to the size of solvent molecules. Since the pressure-denatured structure is characterized by small $v$, it should possess considerably large $a$ despite sufficiently small $v_{\mathrm{ex}}$.

\section{Comparison between calculated and experimentally measured values of partial molar volume}

First, lysozyme (PDB code: 1HEL) is chosen and the EV and PMV are calculated using our simplified model. The solvent number density in the bulk is set at the value for water at $298 \mathrm{~K}$. The PMV obtained is $11600 \mathrm{~cm}^{3} / \mathrm{mol}$ that is in good agreement with the average of the experimentally measured values, ${ }^{27} 10100 \mathrm{~cm}^{3} / \mathrm{mol}$. The EV $\left(\sim 15000 \mathrm{~cm}^{3} / \mathrm{mol}\right)$ is much larger than the PMV and the 
TABLE I. Calculated and experimental values of the partial molar volume (PMV) for five different proteins. The unit is $\mathrm{cm}^{3} / \mathrm{mol}$.

\begin{tabular}{lccc}
\hline \hline Protein & PDB ID & Calc. value & Expt. value $^{\mathrm{a}}$ \\
\hline BPTI & 5PTI & 4870 & 4690 \\
RNase A & 8RAT & 10100 & 9570 \\
Lysozyme & 1HEL & 10600 & 10100 \\
$\beta$-lactoglobulin A & 1BSY & 14400 & 13700 \\
$\alpha$-chymotrypsinogen A & 2CGA & 19700 & 18600 \\
\hline \hline
\end{tabular}

${ }^{\mathrm{a}}$ Reference 27.

parameter $\xi$ in Eq. (3) is certainly positive. The agreement becomes even better when the hard-sphere diameter of each atom in a protein is set at the distance where the LennardJones potential energy equals the thermal energy $k_{B} T$ (Ref. 15). The calculated and experimental values for five different proteins are compared in Table I, which indicates remarkably good agreement despite the simplicity of our model. This result can be explained as follows. It is well known that the water density near a hydrophobic group is lower than the value calculated for our simplified model. When the hydrophobic group is replaced by a hydrophilic one, however, the water density rises to a great extent and becomes higher than the value calculated for our simplified model. Since the exposed surface of a protein comprises both hydrophobic groups and hydrophilic ones which are almost irregularly distributed, ${ }^{12,13,19}$ the cancellation of the overestimation and underestimation occurs. Consequently, the PMV of a protein in aqueous solution can be reproduced fairly accurately by our method even in a quantitative sense, and we can be quite confident in the qualitative aspects of the conclusions drawn in this article.

Furthermore, our method is capable of capturing the high sensitivity of the PMV to the atomic details of protein structure. Two structures at low (PDB code: 1GXV) and high (PDB code: 1GXX) pressures, respectively, have been reported for lysozyme. ${ }^{28}$ These structures are almost indistinguishable by sight, as illustrated in Fig. 2, because the pres-
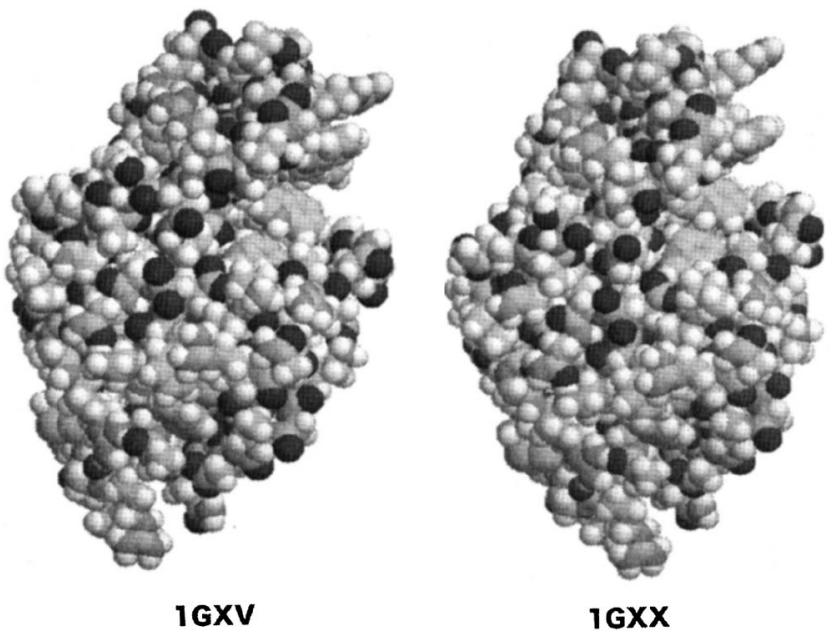

FIG. 2. Space-filled representation of the low-pressure (PDB code: 1GXV) and high-pressure (PDB code: 1GXX) structures of lysozyme.
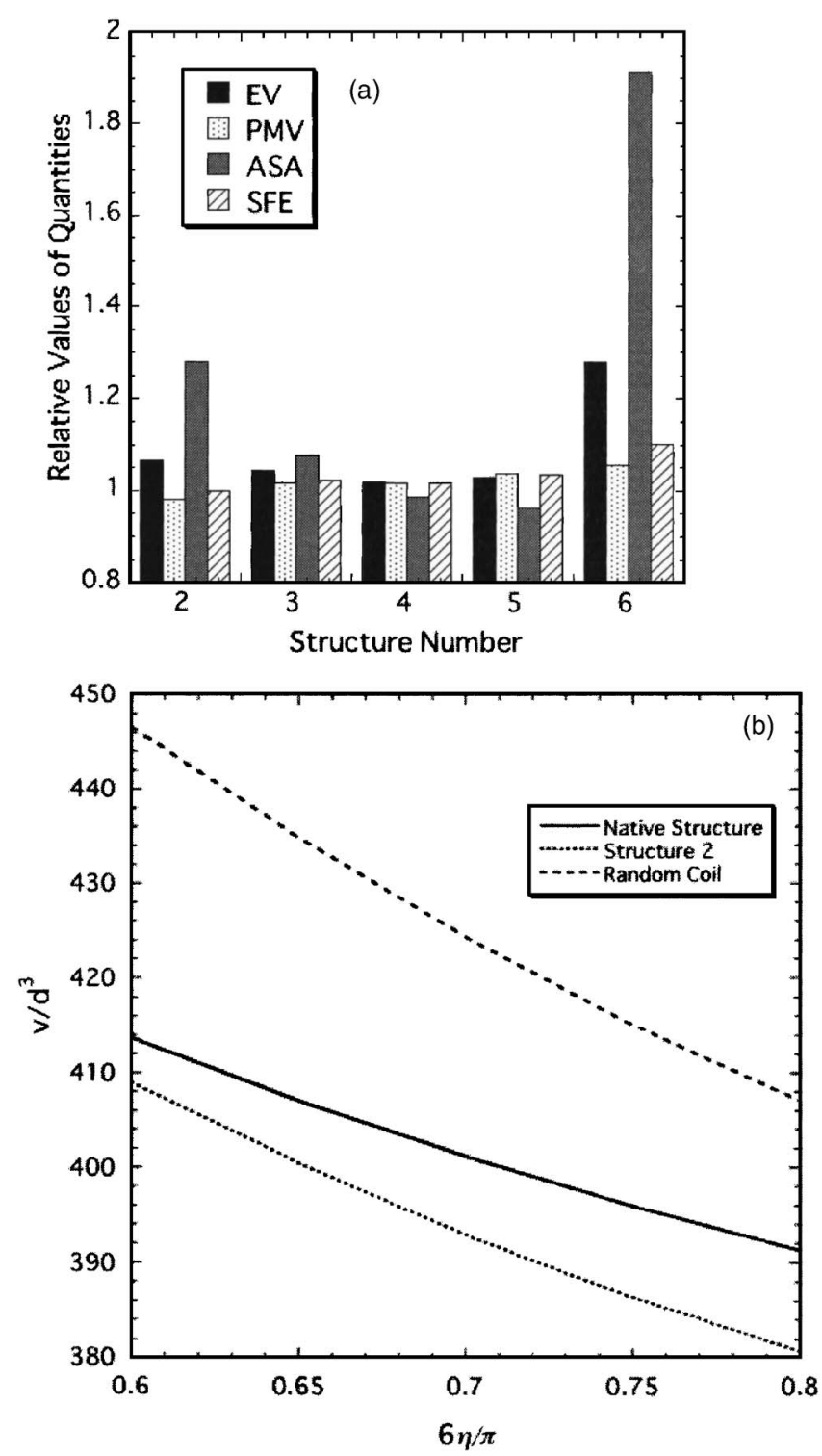

FIG. 3. (a) Values of the excluded volume (EV), partial molar volume (PMV), accessible surface area (ASA), and solvation free energy (SFE) for five representative structures (structures 2-6) of protein G. They are divided by the values for the native structure (structure 1), respectively, and the quotients are referred to as "relative values." Structure 6 is a random coil. Structures 2-5 are much more compact than structure 6 . The diameter of solvent molecules $d$ is $0.28 \mathrm{~nm}$. (b) PMV scaled by $d^{3}$ plotted against $6 \eta / \pi$ ( $\eta$ is the solvent packing fraction) corresponding to the pressure $P$. Structures 1,2 , and 6 are considered. The diameter of solvent molecules $d$ is $0.28 \mathrm{~nm}$.

sure is not high enough to cause appreciable denaturation. Nevertheless, the PMV calculated for the high-pressure structure is smaller than that for the low-pressure one by $\sim 0.9 \%$ at the same solvent density, which is physically reasonable. The high-pressure structure corresponds to structure $\mathrm{Y}$ in Fig. 1. The low- and high-pressure structures share the same ASA, but the EV of the high-pressure structure is slightly smaller. This is suggestive that the void spaces in the protein interior are reduced by applying the moderately high pressure. As discussed below, the pressure-denatured structure shown in Fig. 1 has considerably smaller PMV for a different reason. 

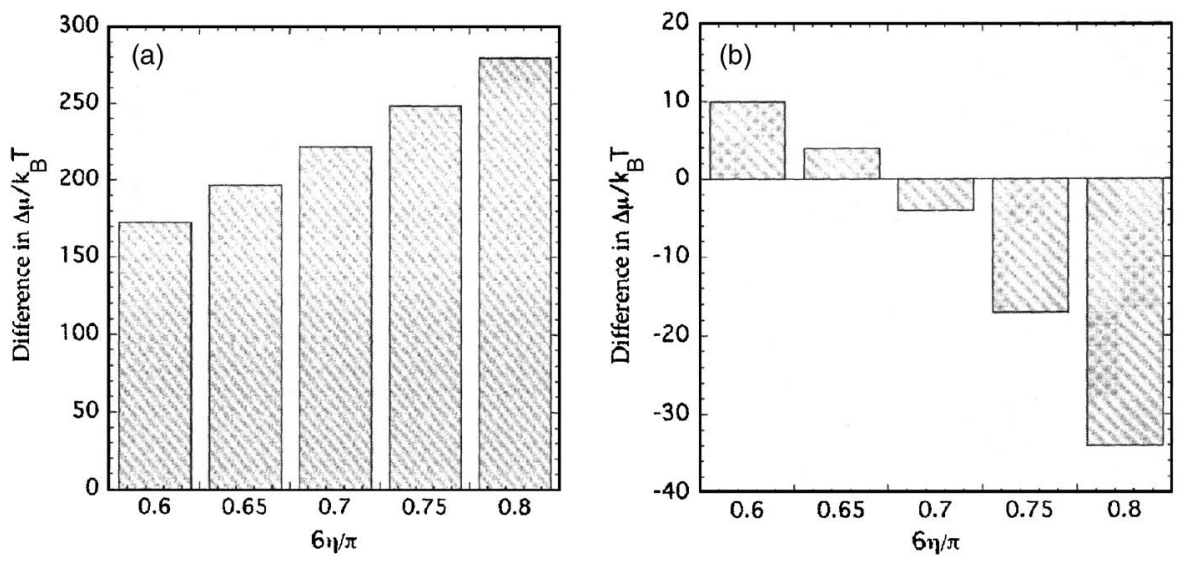

FIG. 4. (a) "Solvation free energy scaled by $k_{B} T\left(\Delta \mu / k_{B} T\right)$ of the random coil minus that of the native structure" plotted against $6 \eta / \pi$ ( $\eta$ is the solvent packing fraction) corresponding to the pressure $P$. The diameter of solvent molecules $d$ is $0.28 \mathrm{~nm}$. (b) " $\Delta \mu / k_{B} T$ of structure 2 minus that of the native structure" plotted against $6 \eta / \pi$. The diameter of solvent molecules $d$ is $0.28 \mathrm{~nm}$.

\section{RESULTS AND DISCUSSION}

\section{A. Pressure dependence of solvation free energy and partial molar volume}

First, we consider six different structures of protein $G$ with 56 residues including the native structure and a random coil. The native structure is taken from the Protein Data Bank (PDB code: 2GB1). The random-coil structure is generated in accordance with the procedure described in the Appendix. The other four structures are taken from the localminimum states of the energy function found in a replicaexchange molecular dynamics simulation using all-atom potentials. ${ }^{29}$ All we wish to do is to analyze some realistic structures of protein $\mathrm{G}$ and clarify the qualitative aspects of the characteristics of pressure-denatured structures. We generate the structures using the computer simulation just to avoid unrealistic overlaps of the polypeptide chain and energetically unreasonable structures. As mentioned above, the pressure-denatured structures are expected to be characterized by small EV, large ASA, and small PMV. Therefore, we have checked the EV, ASA, and PMV of all the structures in the simulation trajectory and attempted to include those structures in our analysis.

Figure 3(a) compares the values of the EV, PMV, ASA, and SFE for the five structures divided by the values for the native structure, respectively $\left(6 \eta / \pi=0.7\right.$, where $\eta=\pi \rho d^{3} / 6$ denotes the solvent packing fraction and corresponds to $P$ ). Structure 1 is the native structure and structure 6 is a random coil. It is observed that the EV and ASA vary greatly from structure to structure while the variation of the PMV and SFE is much smaller. The random coil features extremely large EV and ASA. The EV of the native structure is the smallest. There is only one structure, structure 2, the PMV of which is smaller than that of the native structure. Among the six structures considered, structure 2 has the second smallest value of the EV and the second largest value of the ASA. Hereafter, we focus on structures 1, 2, and 6. In Fig. 3(b) the PMV scaled by $d^{3}$ is plotted against $6 \eta / \pi$. The solvent layer near the protein surface becomes even denser as $P$ increases due to a stronger packing force, and the parameter $\xi$ is a monotonically increasing function of $P$. Hence, the slope of each curve in Fig. 3(b), which is given by

$$
\begin{aligned}
(\partial v / \partial P)_{T} & \sim-(\partial \xi / \partial P)_{T} d a \\
& =-(\partial \xi / \partial \eta)_{T}(\partial \eta / \partial P)_{T} d a, \quad(\partial \eta / \partial P)_{T}>0,
\end{aligned}
$$

is negative and steeper for a structure with larger ASA $\left[(\partial \eta / \partial P)_{T}\right.$ is independent of the protein structure $]$. A significant point is that the PMV of the random coil is larger than that of the native structure while the PMV of structure 2 is smaller. Figure 4(a) shows "the SFE scaled by $k_{B} T\left(\Delta \mu / k_{B} T\right)$ of the random coil minus that of the native structure" plotted against $6 \eta / \pi$ corresponding to $P$. As $P$ increases, the difference becomes larger and the random coil is relatively more destabilized. By contrast, as shown in Fig. 4(b) where " $\left(\Delta \mu / k_{B} T\right)$ of structure 2 minus that of the native structure" is plotted against $6 \eta / \pi$, the inversion of the SFE occurs at a sufficiently high pressure and structure 2 becomes more stable than the native structure. This result is in accord with the physical picture illustrated in Fig. 1 and thermodynamically consistent.

\section{B. Characteristics of pressure-denatured structure}

The six structures are compared in Fig. 5. The random coil has quite large ASA, as observed in Fig. 3(a). However, its EV is extremely large and the PMV remains considerably larger than that of the native structure. The difference be-

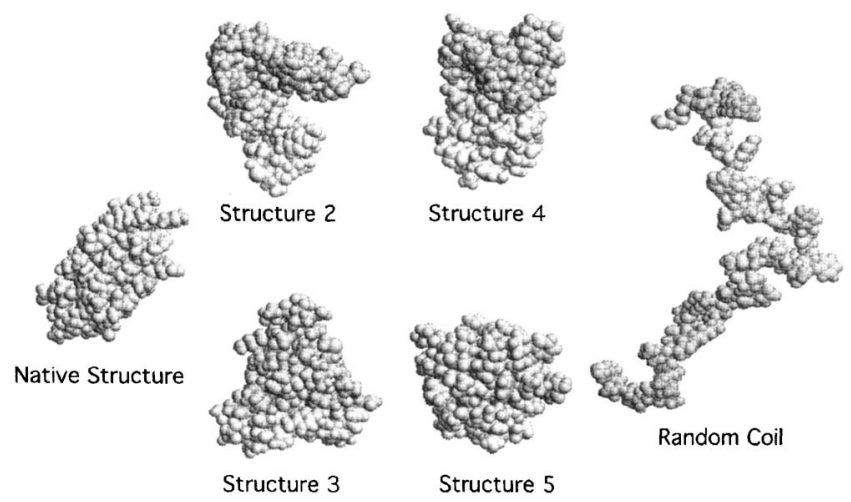

FIG. 5. Space-filled representation of the native structure (structure 1), structures $2-5$, and the random coil (structure 6) of protein G. The native structure has one $\alpha$ helix and one $\beta$ sheet. Structure 3 has four $\alpha$ helices. In structure 4 , the $\alpha$ helix in agreement with the native structure is formed but the $\beta$ sheet is absent. Structure 5 is nearly spherical. Refer to our earlier work (Ref. 13) for more details of structures 3-5. 


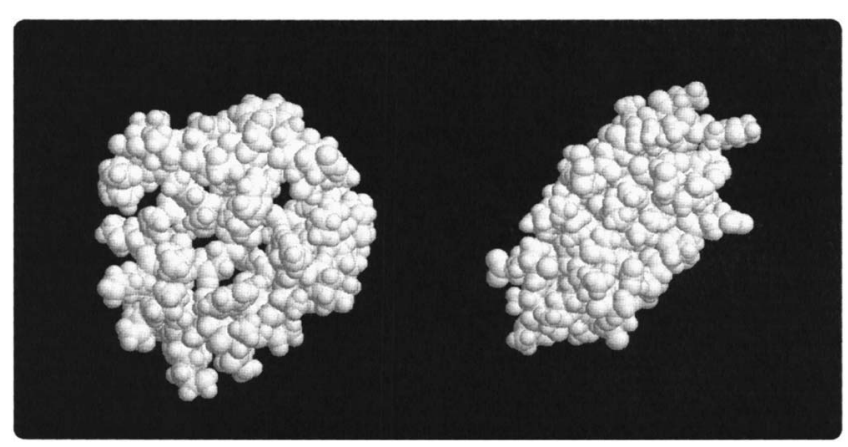

FIG. 6. Space-filled representation of a structure featuring the swelling (left) and the native structure (right) of protein $\mathrm{G}$.

tween the random coil and the native structure in terms of the stability becomes increasingly larger with rising pressure, and the pressure-denatured structure can hardly be like the random coil. Structure 2 has a large cleft allowing the penetration of solvent molecules. This penetration enlarges the ASA while the EV remains sufficiently small. These features are not found in structures 3-5. The pressure-denatured structure should be qualitatively similar to structure 2 . It is only moderately less compact than the native structure; nevertheless its ASA is much larger.

We have chosen several representative structures and analyzed the stability of each structure at different pressures. For a complete argument, structural ensembles of protein states are to be considered. However, the qualitative aspects of the conclusions are not altered. For example, a total of ten different random coils are generated (see the Appendix) but they all share qualitatively the same characteristics. The PMV is always larger than that of the native structure. The average value of the PMV for the ten random coils is larger than that of the native structure by $\sim 2.3 \%$. (The PMV of a fully extended structure is larger by $\sim 6.2 \%$.) In the computer simulations using all-atom potentials, ${ }^{29}$ we also find some more structures whose PMV is smaller than that of the native structure. All of them have small EV and very large ASA and are characterized by the cleft and/or swelling that allows the solvent penetration into the interior. A representative structure featuring the swelling is shown in Fig. 6 where the native structure is also included for comparison. The PMV of the representative structure is smaller than that of the native structure by $\sim 4.2 \%$ and the PMV of structure 2 is smaller by $\sim 2.1 \%$.

The penetration of the solvent molecules into the protein interior can be verified by investigating the reduced solventdensity profile $g(x, y, z)$. Figure 7 shows $g(x, y, z)$ along two example lines for the structure featuring the swelling (how to choose the origin of the coordinate system is described in the Appendix). At the separations which are sufficiently far from the protein surface, $g$ is unity. Near the surface, $g$ is oscillatory and takes a rather large value at contact. In the region where no solvent molecule can be present, $g$ is zero. The penetration of the solvent molecules into a narrow, confined space in the interior is characterized by very sharp peaks of $g$. The figure certainly indicates this type of penetration.

The results described above are in qualitatively good accord with the experimental observations for pressure
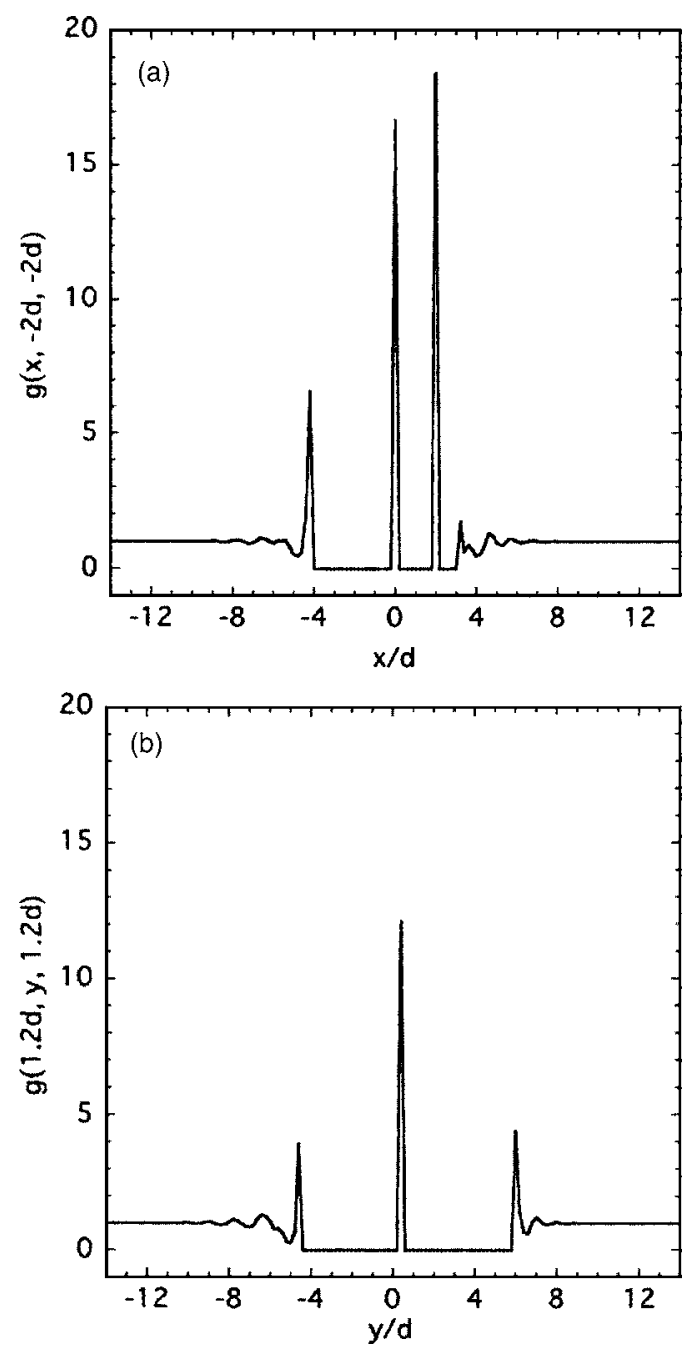

FIG. 7. Reduced solvent-density profiles $(6 \eta / \pi=0.8)$ along two example lines for the structure featuring the swelling shown in Fig. 6. The very sharp peaks in the interior indicate the solvent penetration. (a) $g(x,-2 d,-2 d)$. (b) $g(1.2 d, y, 1.2 d)$

denaturation $^{2-7}$ indicating the swelling, water penetration into the interior, and only a moderate reduction of the compactness. They are also consistent with the results of the theoretical and computer simulation studies. ${ }^{7,9-11}$ It can be concluded that structure 2 and the structure shown in the left side of Fig. 6 well represent the qualitative characteristics of the structures stabilized at high pressures.

The entropically induced denaturation can be interpreted as follows. The presence of a water molecule generates an excluded volume for the other water molecules. This water crowding becomes serious when the pressure is highly increased. The only compromise is the penetration of water molecules into the protein interior for repacking protein atoms with these water molecules even more closely. This relaxes the restriction of the translational movement of the water molecules outside the protein while that of the water molecules in the interior is largely restricted. The former effect dominates at a sufficiently high pressure. The total entropy of water becomes higher when the protein is denatured in the manner described above. 


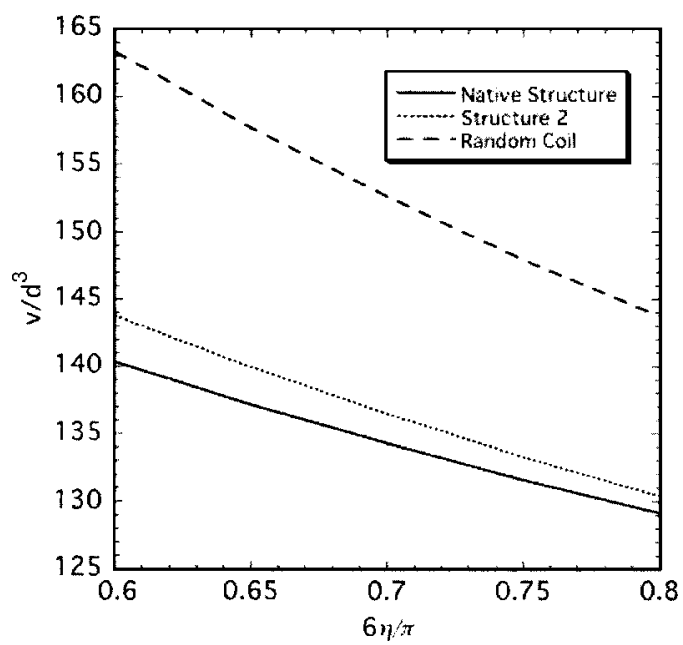

FIG. 8. Partial molar volume (PMV) scaled by $d^{3}$ plotted against $6 \eta / \pi$ ( $\eta$ is the solvent packing fraction) corresponding to the pressure $P$. Structures 1,2, and 6 are considered. The diameter of solvent molecules $d$ is $0.42 \mathrm{~nm}$.

\section{Effects due to size of solvent molecules}

We now increase the diameter of solvent molecules by $50 \%$ and set $d$ at $0.42 \mathrm{~nm}$. Structures 1, 2, and 6 are chosen for the analysis. In Fig. 8 the PMV scaled by $d^{3}$ is plotted against $6 \eta / \pi$. The PMV of structure 2 is always larger than that of the native structure while the opposite is true in the case of $d=0.28 \mathrm{~nm}$ shown in Fig. 3(b). Figure 9(a) shows " $\Delta \mu / k_{B} T$ of the random coil minus that of the native structure" plotted against $6 \eta / \pi$ corresponding to $P$. As $P$ increases, the difference in the SFE becomes larger and the random coil is relatively more destabilized. The same behavior of the pressure dependence is also observed in Fig. 4(a). A more interesting result is shown in Fig. 9(b) where " $\left(\Delta \mu / k_{B} T\right)$ of structure 2 minus that of the native structure" is plotted against $6 \eta / \pi$. The SFE difference increases and structure 2 is relatively more destabilized with rising pressure, which is the opposite of the result shown in Fig. 4(b). Structure 2 is no more a candidate for the pressure-denatured structures if it is immersed in the solvent with the larger molecular size.

We now discuss why the PMV of structure 2 becomes larger than that of the native structure for the larger solvent diameter. Table II compares the EV, ASA, PMV, and parameter $\xi$ in the two cases, $d=0.28 \mathrm{~nm}$ and $d=0.42 \mathrm{~nm}$. Both of the EV and ASA increase for the native structure and structure 2 when the solvent diameter becomes larger, which can readily be expected from the definition of the EV and ASA. Here, we define the quantities $v_{\mathrm{ex}}^{*}$ and $a^{*}$ by

$$
v_{\mathrm{ex}}^{*}=v_{\mathrm{ex}}-v_{0}
$$

and

$$
a^{*}=a-a_{0},
$$

respectively. In Eqs. (5), $v_{0}$ and $a_{0}$ denote the volume and surface area of the protein molecule itself and are independent of the protein structure. For a hard-sphere solute with diameter $D(D \gg d), v_{\mathrm{ex}}^{*}=\pi(D+d)^{3} / 6-\pi D^{3} / 6 \sim \pi D^{2} d / 2$ and $a^{*}=\pi(D+d)^{2}-\pi D^{2} \sim 2 \pi D d: v_{\mathrm{ex}}^{*} / d$ and $a^{*} / d$ are constant
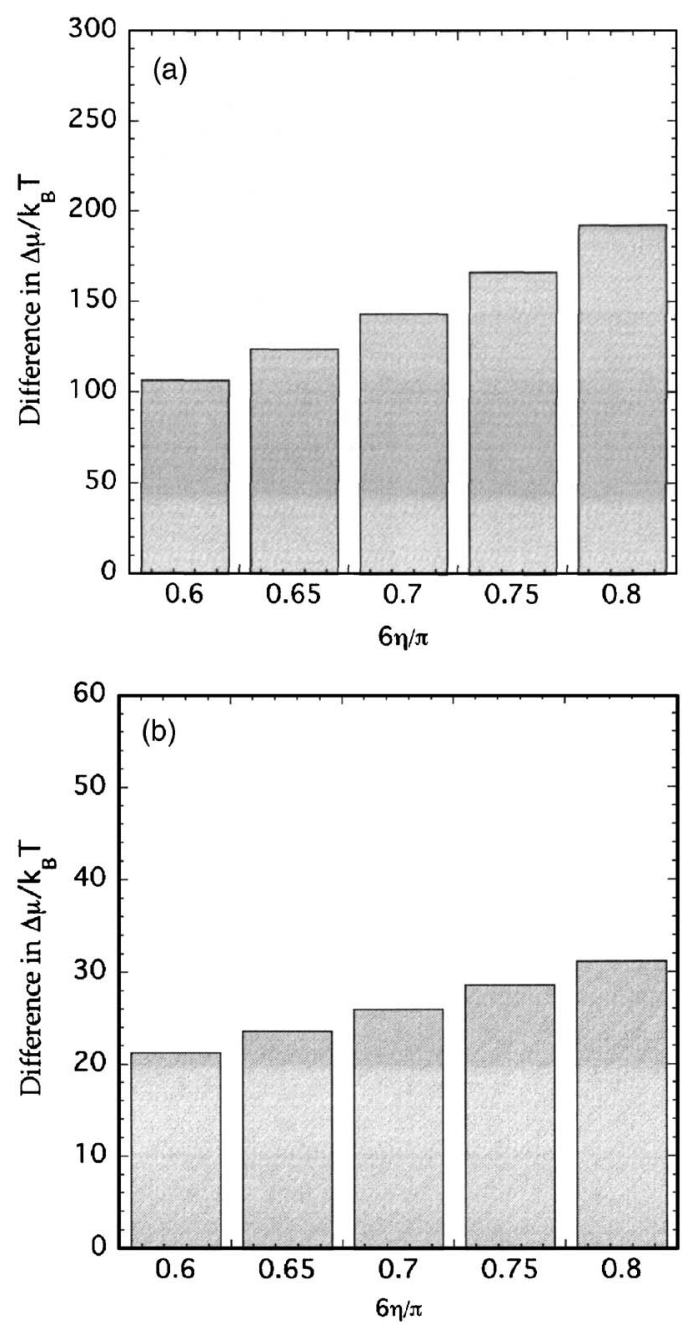

FIG. 9. (a) "Solvation free energy scaled by $k_{B} T\left(\Delta \mu / k_{B} T\right)$ of the random coil minus that of the native structure" plotted against $6 \eta / \pi$ ( $\eta$ is the solvent packing fraction) corresponding to the pressure $P$. The diameter of solvent molecules $d$ is $0.42 \mathrm{~nm}$. (b) " $\Delta \mu / k_{B} T$ of structure 2 minus that of the native structure" plotted against $6 \eta / \pi$. The diameter of solvent molecules $d$ is $0.42 \mathrm{~nm}$.

against the change in the solvent diameter. By contrast, for a protein with the complex polyatomic structure, $v_{\mathrm{ex}}^{*} / d$ and $a^{*} / d$ are dependent on the geometric features of the structure and the solvent diameter. In other words, when the solventdiameter dependence is discussed, these parameters are

TABLE II. The excluded volume (EV), accessible surface area (ASA), partial molar volume (PMV), and parameter $\xi$ calculated for structure 1,2, and 6 of protein $\mathrm{G}$ in two solvents with different molecular diameters. Structure 1 is the native structure and structure 6 is the random coil.

\begin{tabular}{crrrr}
\hline \hline Structure & $v_{\text {ex }}\left(\mathrm{nm}^{3}\right)$ & $A\left(\mathrm{~nm}^{3}\right)$ & $v\left(\mathrm{~nm}^{3}\right)$ & $\xi(-)$ \\
\hline & & $d=0.28 \mathrm{~nm}$ & & \\
1 & 11.98 & 39.26 & 8.802 & 0.289 \\
2 & 12.79 & 50.26 & 8.624 & 0.296 \\
6 & 15.33 & 75.06 & 9.312 & 0.286 \\
& & $d=0.42 \mathrm{~nm}$ & & \\
1 & 14.76 & 40.31 & 9.948 & 0.284 \\
2 & 16.30 & 50.44 & 10.11 & 0.292 \\
6 & 20.73 & 79.02 & 11.30 & 0.284 \\
\hline \hline
\end{tabular}


TABLE III. Difference between structures 1 and 2 in the excluded volume (EV) and accessible surface area (ASA) scaled by the solvent diameter. Two solvent with different molecular diameters are considered. Structure 1 is the native structure.

\begin{tabular}{ccc}
\hline \hline & $d=0.28(\mathrm{~nm})$ & $D=0.42(\mathrm{~nm})$ \\
\hline$\left(v_{\mathrm{ex}, 2}^{*}-v_{\mathrm{ex}, 1}^{*}\right) / d\left(\mathrm{~nm}^{2}\right)$ & 2.89 & 3.67 \\
$\left(a_{2}^{*}-a_{1}^{*}\right) / d(\mathrm{~nm})$ & 39.3 & 24.1 \\
\hline \hline
\end{tabular}

good measures of the EV and ASA which in effect influence the PMV. Let $v_{\mathrm{ex}, J}^{*}$ and $a_{J}^{*}$ denote $v_{\mathrm{ex}}^{*}$ and $a^{*}$ of structure $J$, respectively. Table III compares $\left(v_{\mathrm{ex}, 2}^{*}-v_{\mathrm{ex}, 1}^{*}\right) / d$ and $\left(a_{2}^{*}-a_{1}^{*}\right) / d$ in the two cases, $d=0.28 \mathrm{~nm}$ and $d=0.42 \mathrm{~nm}$. As observed in Table III, when $d$ increases from 0.28 to $0.42 \mathrm{~nm},\left(v_{\mathrm{ex}, 2}^{*}-v_{\mathrm{ex}, 1}^{*}\right) / d$ increases while $\left(a_{2}^{*}-a_{1}^{*}\right) / d$ exhibits a remarkable decrease (they increase and decrease by $\sim 27 \%$ and $\sim 39 \%$, respectively). This result implies that for a larger value of $d$ the EV and ASA of structure 2 virtually become larger and smaller, respectively, as compared to those of the native structure. The most important result shown in Table II is that in the case of $d=0.42 \mathrm{~nm}$ the PMV of structure 2 is no longer smaller than that of the native structure. This is mainly ascribed to the virtual decrease in the ASA and increase in the EV (we remark that the parameter $\xi$ is only slightly smaller for $d=0.42 \mathrm{~nm}$ than for $d=0.28 \mathrm{~nm}$ ).

The above result, which is suggestive that pressure denaturation is less likely to occur in the solvent with a larger molecular size, can be understood in the following way. Large ASA of the pressure-denatured structure is ascribed to water penetration into the protein interior. Since the molecular size of water is exceptionally small, the resultant structure remains fairly compact despite the penetration. The EV of the denatured structure is only slightly larger than that of the native structure with the result that $\xi$ and the ASA are large enough to make the PMV smaller. If the penetration of solvent molecules with a considerably larger size occurs, however, not only the ASA increases but also the compactness is significantly vitiated. The resultant structure has much larger EV, leading to large PMV. When the molecular size of the solvent is too large, there can be no structure whose PMV is smaller than that of the native structure and the denaturation always leads to a loss of the solvent entropy. The exceptionally small molecular size of water enables a protein to remain sufficiently compact and to expose a large portion of its surface to water at the same time. We have thus provided a physical interpretation of the experimentally inferred result that water is crucial for pressure denaturation. ${ }^{8}$

\section{SUMMARY AND CONCLUSIONS}

It was suggested that the entropic effect arising from the translational movement of water molecules plays crucially important roles in a variety of processes occurring in biological systems. ${ }^{12,13,23,24,30-34}$ Let us emphasize that recent experiments have shown that the amyloid-fibril formation, ${ }^{35}$ the lock-key interaction, ${ }^{36}$ and the association of virus ${ }^{37}$ are entropically driven at ambient pressures. At the same time, it has been shown in experiments that high pressures reverse the aggregation of protein folding intermediates ${ }^{38}$ and dissociate the amyloid fibrils ${ }^{39}$ or the multiprotein virus assemblies. ${ }^{37}$ We believe that the phenomena occurring at the high pressures are also entropically driven. As a striking example, we have treated the folding/unfolding transition of proteins and shown that pressure denaturation can be elucidated in terms of the purely entropic effect and the exceptionally small size of water molecules is crucial in the denaturation.

In our statistical-mechanical method, the protein structure is characterized by the $\mathrm{EV}$ for the solvent molecules and the ASA. The PMV, the pressure derivative of the SFE, is expressed in terms of the EV, ASA, and parameter $\xi$ related to the solvent-density profile entropically formed near the protein surface. By a thermodynamic argument combined with our method, the denatured structures are shown to have only moderately larger EV, much larger ASA, and sufficiently smaller PMV as compared to the native one and to turn more stable at an elevated pressure. They are characterized by the cleft and/or swelling, solvent penetration into the interior, and only a moderate reduction of the compactness. This result is consistent with the experimental observations. $^{2-7}$

The entropically driven pressure denaturation can be interpreted in words as follows. A protein is driven to take a structure maximizing the translational entropy of water. At a low pressure, the native structure is greatly stabilized by the entropic effect. When a moderately high pressure is applied, the void spaces in the interior are reduced with the native structure preserved. At an even higher pressure, the water crowding is quite serious but it is almost impossible to further reduce the void spaces. (We note that the presence of a water molecule generates an excluded volume for the other water molecules.) The only compromise is the penetration of water molecules into the protein interior for repacking protein atoms with these water molecules even more closely. This relaxes the restriction of the translational movement of the water molecules outside the protein while that of the water molecules in the interior is largely restricted. The former effect dominates at a sufficiently high pressure. The total entropy of water becomes higher when the protein is denatured. It is quite interesting that the entropic effect, which greatly stabilizes the native structure at a low pressure, drives the protein to be denatured at a sufficiently high pressure. The gain or loss of the translational entropy of water upon the structural change of a protein must be considered in terms of all the water molecules that are present in the system. This is in contrast with the gain or loss of the rotational entropy which originates mainly from the water molecules adjacent to the protein surface.

At high pressures, even a small change of the protein structure causes a large increase or decrease in the water entropy, and differences in the stability among the structures are remarkably magnified. Hence, in the high-pressure region, it is rather questionable if many different structures are almost equally stable at a given pressure. This is in contrast with the case of the heat-denatured structures at low pressures. The entropic effect discussed so far is a major cause of the solute hydrophobicity. At a higher pressure the hydropho- 
bicity is strengthened in the sense that the water crowding is serious and the hydration free energy becomes higher. The previously suggested concept, ${ }^{11}$ the weakening of the hydrophobic interaction between nonpolar side chains is responsible for pressure denaturation (see the third paragraph in Sec. I), is inconsistent with our view.

When the size of solvent molecules is set 1.5 times larger than that of water molecules, the inversion of the stability mentioned above is no more observed. In the solvent with a larger molecular size, pressure denaturation is less likely to take place. In water, water penetration into the protein interior leads to a large increase in the ASA, but the compactness of the structure is almost preserved due to the very small size of water molecules. However, if the penetration of solvent molecules with a considerably larger size occurs, not only the ASA increases but also the compactness is significantly vitiated. When the size is too large, there can be no structure whose PMV is smaller than that of the native structure and the denaturation always leads to a loss of the solvent entropy. It was inferred from experimental results that water is crucial for pressure denaturation. ${ }^{8}$ Our theoretical analysis has supported this inference with the physical interpretation that the exceptionally small molecular size of water is responsible for the crucial importance of water in pressure denaturation.

In the Asakura-Oosawa theory, ${ }^{40,41}$ a conventional theory for considering the entropic effect, the formation of the solvent microstructure near a protein is neglected with the result that the PMV always equals the EV. The native structure is even more stabilized than any less compact one with larger $\mathrm{EV}$ as the pressure increases. By contrast, this drawback is not inherent in the 3D integral equation theory ${ }^{12,13,23,24}$ we have employed. The theory, which also allows us to analyze the interaction entropically induced between biomolecules, ${ }^{24,30}$ provides the possibility to study the pressure effects on various physicochemical processes occurring in aqueous solutions and biological systems. For example, the high pressure reverses the aggregation of protein folding intermediates $^{38}$ and dissociates the amyloid fibrils ${ }^{39}$ or the multiprotein virus assemblies, ${ }^{37}$ which is to be tackled in future studies.

\section{ACKNOWLEDGMENTS}

This work was supported by Grants-in-Aid for Scientific Research on Priority Areas (Grant No. 15076203) from the Ministry of Education, Culture, Sports, Science and Technology of Japan and by NAREGI Nanoscience Project.

\section{APPENDIX: THEORETICAL METHOD}

The integral equation theory is a statistical-mechanical theory which is widely used in liquid state physics. ${ }^{42}$ It was originally developed for a spherically symmetric system. The $3 \mathrm{D}$ integral equation theory we employ is an extension to general systems described using the $x-y-z$ coordinate system. The great advantage of the $3 \mathrm{D}$ version is that details of the polyatomic structure of a solute molecule can explicitly be taken into account. Similar approaches were employed by several authors ${ }^{22,43-46}$ to analyze the solvation properties of a solute molecule. In our case, the 3D integral equation theory is applied to the special model system described below.

Solute $I$, a protein molecule with a prescribed conformation, is immersed in small spheres forming the solvent at infinite dilution. Solute $I$ consists of a set of fused atoms. In the 3D integral equation theory, the Ornstein-Zernike (OZ)

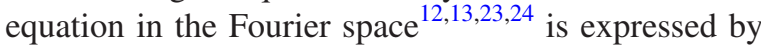

$$
W_{I S}\left(k_{x}, k_{y}, k_{z}\right)=\rho_{S} C_{I S}\left(k_{x}, k_{y}, k_{z}\right) H_{S S}(k),
$$

and the closure equation ${ }^{12,13,23,24}$ is written as

$$
\begin{aligned}
c_{I S}(x, y, z)= & \exp \left\{-u_{I S}(x, y, z) /\left(k_{B} T\right)\right\} \exp \left\{w_{I S}(x, y, z)\right. \\
& \left.+b_{I S}(x, y, z)\right\}-w_{I S}(x, y, z)-1 .
\end{aligned}
$$

Here, the subscript $S$ denotes the solvent, $c$ is the direct correlation function, $h$ is the total correlation function, $w=h-c$, $u$ is the spatial distribution of the solvent-solute potential, $k_{B} T$ is Boltzmann's constant times the absolute temperature, and $\rho_{S}$ is the solvent number density. The capital letters $(C$, $H$, and $W)$ represent the Fourier transforms. $H_{S S}(k)\left(k^{2}=k_{x}^{2}\right.$ $\left.+k_{y}^{2}+k_{z}^{2}\right)$ is calculated using the integral equation theory for spherical particles and served as part of the input data. In the hypernetted-chain (HNC) approximation employed in the present study, the bridge function $b$ is set at zero. The reliability of the HNC closure equation has already been verified. $^{23,47}$

Equations (A1) and (A2) are numerically solved on a cubic grid. The $x-y-z$ coordinates of the protein atoms in the native structure are taken from the Protein Data Bank (PDB). As for the protein atoms in the random coil, the coordinates are obtained in the following manner. First, a conformation is generated by randomly assigning dihedral angles of the backbone. Second, in order to eliminate all the unreasonable overlaps, the constituent atoms are moved to the locally optimized coordinates by employing a standard energyminimization method with the all-atom potentials. The center of the protein molecule $\left(x_{C}, y_{C}, z_{C}\right)$ is calculated from

$$
x_{C}=\sum_{i=1}^{M} x_{i} / M, \quad y_{C}=\sum_{i=1}^{M} y_{i} / M, \quad z_{C}=\sum_{i=1}^{M} z_{i} / M,
$$

where $M$ denotes the total number of the atoms. The center is then chosen as the origin of the coordinate system and the $x-y-z$ coordinates of the protein atoms are recalculated as $\left(x_{i}-x_{C}, y_{i}-y_{C}, z_{i}-z_{C}\right) \quad(i=1, \ldots, M)$. The numerical procedure is briefly summarized as follows.

(1) Calculate $u_{I S}(x, y, z)$ at each 3D grid point.

(2) Initialize $w_{I S}(x, y, z)$ to zero.

(3) Calculate $c_{I S}(x, y, z)$ using Eq. (A2).

(4) Transform $c_{I S}(x, y, z)$ to $C_{I S}\left(k_{x}, k_{y}, k_{z}\right)$ using the $3 \mathrm{D}$ fast Fourier transform (3D-FFT).

(5) Calculate $W_{I S}\left(k_{x}, k_{y}, k_{z}\right)$ from Eq. (A1).

(6) Invert $W_{I S}\left(k_{x}, k_{y}, k_{z}\right)$ to $w_{I S}(x, y, z)$ using the 3D-FFT.

(7) Repeat steps (3)-(6) until the input and output functions become identical within convergence tolerance.

The solvent molecules are modeled as hard spheres and solute $I$ is treated as a set of fused hard spheres. On grid points where the solvent particle and at least one of the atoms over- 
lap, $\exp \left\{-u_{I S}(x, y, z) /\left(k_{B} T\right)\right\}$ is zero. Otherwise, it is unity. The grid spacing $(\Delta x, \Delta y$, and $\Delta z)$ is set at $0.2 d_{S}\left(d_{S}\right.$ is the solvent diameter) and the grid resolution $\left(N_{x} \times N_{y} \times N_{z}\right)$ is $256 \times 256 \times 256$. It has been verified that the spacing is sufficiently small and the box size $\left(N_{x} \Delta x, N_{y} \Delta y\right.$, and $\left.N_{z} \Delta z\right)$ is large enough.

The density structure of the solvent near solute $I$ is obtained as $g_{I S}(x, y, z)(g=h+1)$. A great advantage of our theory is that the solvation free energy (SFE) of solute $I$, $\Delta \mu_{I}$, is obtained from the simple integration of the direct and total correlation functions ${ }^{12,13,24}$ expressed by

$$
\begin{aligned}
\Delta \mu_{I} /\left(k_{B} T\right)= & \rho_{S} \iiint\left\{h_{I S}(x, y, z)^{2} / 2-c_{I S}(x, y, z)\right. \\
& \left.-h_{I S}(x, y, z) c_{I S}(x, y, z) / 2\right\} d x d y d z .
\end{aligned}
$$

In the text, $\rho_{S}, d_{S}, g_{I S}$, and $\Delta \mu_{\mathrm{I}}$ are simply written as $\rho, d, g$, and $\Delta \mu$, respectively.

${ }^{1}$ C. M. Dobson, Nature (London) 426, 884 (2003).

${ }^{2}$ C. A. Royer, Biochim. Biophys. Acta 1595, 201 (2002)

${ }^{3}$ G. Panick, G. J. A. Vidugiris, R. Malessa, G. Rapp, R. Winter, and C. A. Royer, Biochemistry 38, 4157 (1999).

${ }^{4}$ J. Woenckhaus, R. Kohling, P. Thiyagarajan, K. C. Litterell, S. Seifert, C. A. Royer, and R. Winter, Biophys. J. 80, 1518 (2001).

${ }^{5}$ G. S. Chryssomallis, P. M. Torgerson, H. G. Drickamer, and G. Weber, Biochemistry 20, 3955 (1981).

${ }^{6}$ C. Clery, F. Renault, and P. Masson, FEBS Lett. 370, 212 (1995).

${ }^{7}$ A. Paliwal, D. Asthagiri, D. P. Bossev, and M. E. Paulaitis, Biophys. J. 87, 3479 (2004).

${ }^{8}$ A. C. Oliveira, L. P. Gaspar, A. T. Da Poian, and J. L. Silva, J. Mol. Biol. 240, 184 (1994).

${ }^{9}$ G. Hummer, S. Garde, A. E. García, M. E. Paulaitis, and L. R. Pratt, Proc. Natl. Acad. Sci. U.S.A. 95, 1552 (1998)

${ }^{10}$ B. Wroblowski, J. F. Díaz, K. Heremans, and Y. Engelborghs, Proteins: Struct., Funct., Genet. 25, 446 (1996).

${ }^{11}$ D. Pascheck and A. E. García, Phys. Rev. Lett. 93, 238105 (2004).

${ }^{12}$ Y. Harano and M. Kinoshita, Chem. Phys. Lett. 399, 342 (2004).

${ }^{13}$ Y. Harano and M. Kinoshita, Biophys. J. 89, 2701 (2005).

${ }^{14}$ N. M. Cann and G. N. Patey, J. Chem. Phys. 106, 8165 (1997).
${ }^{15}$ T. Imai, Y. Harano, M. Kinoshita, A. Kovalenko, and F. Hirata, J. Chem. Phys. (in press).

${ }^{16}$ T. Lazaridis and M. E. Paulaitis, J. Phys. Chem. 98, 635 (1994).

${ }^{17}$ M. Kinoshita, N. Matubayasi, Y. Harano, and M. Nakahara, J. Chem. Phys. 124, 024512 (2006).

${ }^{18}$ W. Kauzmann, Nature (London) 325, 763 (1987).

${ }^{19}$ C. N. Pace, Biochemistry 40, 310 (2001).

${ }^{20}$ K. Soda, J. Phys. Soc. Jpn. 62, 1782 (1993)

${ }^{21}$ Y. Harano and M. Kinoshita, J. Phys.: Condens. Matter 18, L107 (2006).

${ }^{22}$ M. Ikeguchi and J. Doi, J. Chem. Phys. 103, 5011 (1995).

${ }^{23}$ M. Kinoshita, J. Chem. Phys. 116, 3493 (2002).

${ }^{24}$ M. Kinoshita, Chem. Phys. Lett. 387, 47 (2004).

${ }^{25}$ Y. Harano, T. Imai, A. Kovalenko,M. Kinoshita, and F. Hirata, J. Chem. Phys. 114, 9506 (2001).

${ }^{26}$ M. L. Connolly, J. Appl. Crystallogr. 16, 548 (1983).

${ }^{27}$ T. Imai, A. Kovalenko, and F. Hirata, Chem. Phys. Lett. 395, 1 (2004).

${ }^{28}$ M. Refaee, T. Tezuka, K. Akasaka, and M. P. Williamson, J. Mol. Biol. 327, 857 (2003).

${ }^{29}$ Y. Okamoto, Recent Research Developments in Pure \& Applied Chemistry 2, 1 (1998).

${ }^{30}$ M. Kinoshita, Chem. Phys. Lett. 387, 54 (2004).

${ }^{31}$ M. Kinoshita and T. Oguni, Chem. Phys. Lett. 351, 79 (2002).

${ }^{32}$ R. Roth, R. van Roij, D. Andrienko, K. R. Mecke, and S. Dietrich, Phys. Rev. Lett. 89, 088301 (2002).

${ }^{33}$ R. Roth and D. Gillespie, Phys. Rev. Lett. 95, 247801 (2005).

${ }^{34}$ M. Kinoshita, Chem. Eng. Sci. 61, 2150 (2006).

${ }^{35}$ J. Kardos, K. Yamamoto, K. Hasegawa, H. Naiki, and Y. Goto, J. Biol. Chem. 279, 55308 (2004).

${ }^{36}$ H. Ohtaka, A. Schön, and E. Freire, Biochemistry 42, 13659 (2003).

${ }^{37}$ C. F. Bonafe, C. M. Vital, R. C. Telles, M. C. Gonçalves, M. S. Matsuura, F. B. Pessine, D. R. Freitas, and J. Vega, Biochemistry 37, 11097 (1998).

${ }^{38}$ B. M. Gorovits and P. M. Horowitz, Biochemistry 37, 6132 (1998).

${ }^{39}$ D. Foguel, M. C. Suarez, A. D. Ferrão-Gonzales et al., Proc. Natl. Acad. Sci. U.S.A. 100, 9831 (2003).

${ }^{40} \mathrm{~S}$. Asakura and F. Oosawa, J. Chem. Phys. 22, 1255 (1954).

${ }^{41}$ S. Asakura and F. Oosawa, J. Polym. Sci. 33, 183 (1958).

${ }^{42}$ J.-P. Hansen and I. R. McDonald, Theory of Simple Liquids, 2nd ed. (Academic, London, 1986)

${ }^{43}$ D. Beglov and B. Roux, J. Chem. Phys. 103, 360 (1995).

${ }^{44}$ C. M. Cortis, P. J. Rossky, and R. A. Friesner, J. Chem. Phys. 107, 6400 (1997).

${ }^{45}$ A. Kovalenko and F. Hirata, Chem. Phys. Lett. 290, 237 (1998).

${ }^{46}$ A. Kovalenko, F. Hirata, and M. Kinoshita, J. Chem. Phys. 113, 9830 (2000).

${ }^{47}$ M. Kinoshita, S. Iba, K. Kuwamoto, and M. Harada, J. Chem. Phys. 105, 7177 (1996). 